\title{
Preservation of the Temporal Branch of the Facial Nerve in Pterional-Transzygomatic Craniotomy
}

\author{
M. Ammirati, A. Spallone ${ }^{1}$, J. Ma, M. Cheatham, and D. Becker \\ Division of Neurosurgery, University of Calilotnia, Los Angeles, CA, U.S.A. and ${ }^{1}$ Division of Neurosutgery, University of Rome "Tor \\ Vergata", Rome, Ita]y
}

\begin{abstract}
Summary
The temporal branch of the facial netve may be saved in a pterional-transzygomatic craniotomy if the dissection is carried beneath the superficial temporal-fáscia. The anatonical and technical aspects of this exposure are described in this report
\end{abstract}

Keywords: Surgical technique; facial nerve; pterional-transzygomatic crimiotony.

\section{Introduction}

Soft tissue dissection in the pterional-zygomatic area carrics a significant risk of injuring the temporal branch of the facial nerve with resulting cosmetic deformity due to the loss of function of the frontalis and orbicularis oculi muscles, among others. The facial nerve branches are at risk while traveling in the sub-galeal space. The potential for injury of these branches is maximum in the classical sub-galeal pterional dissection while it is less if the temporal muscle is elevated together with the scalp ${ }^{10,11}$. However, a myocutaneous flap decreases the exposure due to the bulky temporal muscle $^{10,11}$. Yaşargil modified the classical sub-galeal pterional dissection, proposing a dissection between the two layers of the superficial temporal fascia ${ }^{10,11}$. Yat şargil's inter-fascial pterional approach has been described in the context of a pterional craniotomy ${ }^{10, \text { II }}$ In the past few years many modifications of pterional craniotomy to include zygomatic arch resection have been reported ${ }^{1,2,5-8}$. Preservation of the temporal branch of the facial nerve is desirable in these approaches as well.

We have recently described the surgical anatomy of the temporal branch of the facial nerve and have suggested a dissection strategy that seemed anatomically suited to preserve this nerve in pterional or transzygomatic approaches ${ }^{3}$. The present report describes a surgical technique derived from our anatomico-surgical study. The initial results obtained using this dissection technique are also reported.

\section{Anatomical Consideration}

We have described in detail in a previous report the anatomy of the pterional-zygomatic region vis a vis the temporal muscle fasciae, the temporal fat pads and the temporal branch of the facial nerve ${ }^{3}$. Briefly stated the galea from the fronto-temporal region thins out toward the zygomatic arch to blend with the subcutaneous tissue. The superficial temporal fascia, a relatively thick fascial investment of the temporalis muscle, attaches inferiorly to the fronto-zygomatic bone and to the zygomatic arch. The antero-inferior portion of the superficial temporal fascia splits into 2 layers in between which there is some adipose tissue (intrafascial fat pad). Small vessels and, occasionally, a twig of the temporal branch of the facial nerve are also contained in this intrafascial fat pad. The deep temporal fascia, very thin, covers the temporalis muscle. Other authors refer to the galea of the temporal region as to the temporoparietal or superficial temporal fascia and to the superficial temporal fascia as to the deep temporal fascia ${ }^{3}$. In addition to the intrafascial fat pad there are 2 other fat pads in the region. One is found superficially in the subcutaneous tissue (superficial fat pad), while the other one is found beneath the superficial temporal fascia, between this fascia and the temporalis muscle (deep fat pad). The temporal branch of the facial nerve pierces the parotid fascia slightiy anterior to the middle 
wi the rygonitic a reth, always anterior to the superficial temperat ittery stem. From there on this nerve is locattul in the subgaleal space, always superficial to the inperficial temporal fascia ${ }^{3}$.

\section{Surgical Technique}

A bicoronal or a reverse question mark skin incision may be used. The lowermost portion of the incision extends $1 \mathrm{~cm}$ below the zygomatic arch immediately anterior to the tragus. Inferiorly, below the zygomatic arch, the incision is carried only through the skin and subcut, remaining posterior to the superficial temporal artery trunk while superior to the zygomalic arch, the incision is carried to the superficint temporal fascia.

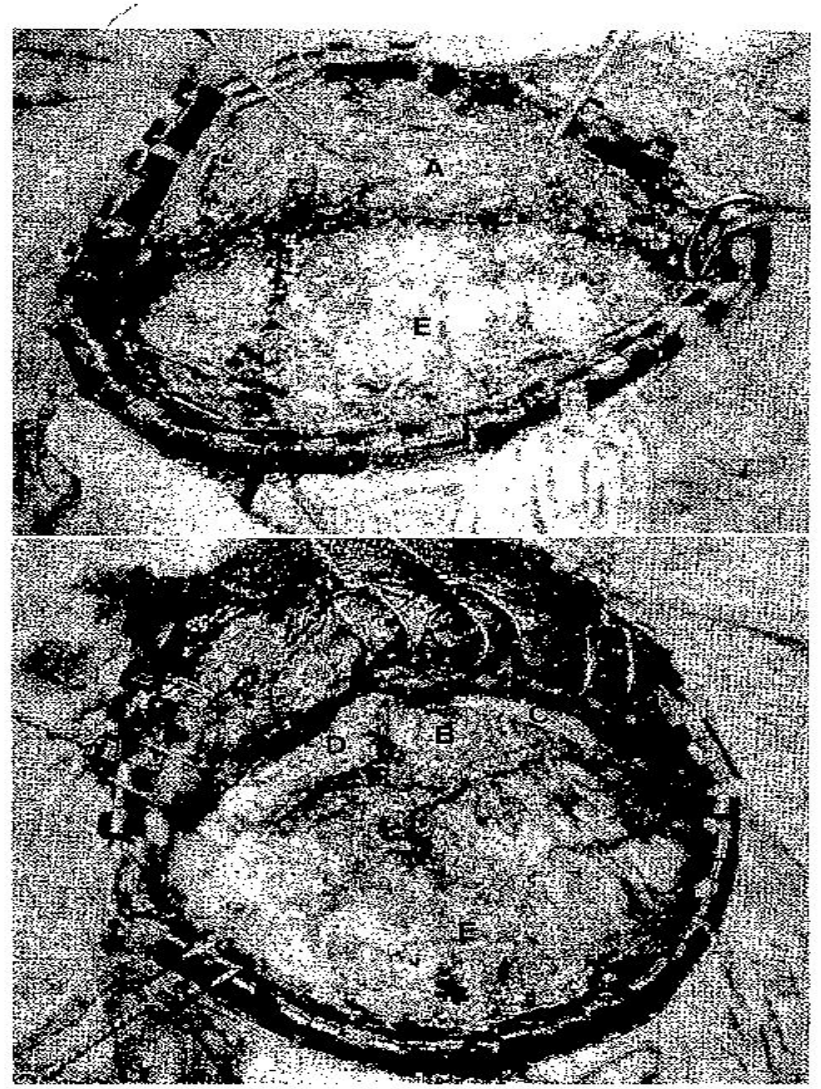

Fig. 1. Intra-operative photograph showing the soft tissue dissection to expose the pterional-zygomatic area. Initial stage (top); final stage (bottom). $A$ superficial temporal fascia; $B$ deep fat pad; $C$ zygomatic arch; $D$ fronto-zygomatic bone; $E$ tempotalis muscle
The superficial temporal fascia is then incised and separated from the temporalis muscle covered by the deep temporal fascia. Inferior to the zygomatic arch the incision is carried deeper to the parotid fascia and is carried only inferiorly enough to have control of the inferior border of the arch (Fig. 1). The dissection is then carried anteriorly, always remaining in this subfascial plane. In doing so one develops a combined scalp and superficial temporal fascia flap that may be extended all the way to the orbit after having released the anterior insertions of the superficial temporal fascia. This flap fully exposes the temporal muscle covered by the deep temporal fascia (Fig. 1). The zygomatic arch may then be transected and the temporal muscle dealt with in the usual way.

\section{Results}

This subfascial approach has been thes! 10 times in various lesions requiring a pterional or plerionaltratnszygomatic craniotony, includirg nooplastic and vascular lesions. There was no evickence of temporal branch paralysis (Fig. 2).

\section{Discussion}

Subgaleal dissection of the plerionil region is associated with a $30 \%$ incidence of tempromal branch palsy $^{10}$. In order to avoid this piltalysis Yaşatgil developed and proposed a dissection rolle between the 2 layers of the superficial temporal fascias" t. His results have not always been reproducible probably due to anatomical vartation in the subgaleal course of the temporal branch of the facial nerve and to difficulties in exactly defining the 2 layers of the superficial temporal fascia ${ }^{3}$. Exposure of the pterional region obtained via a myocutaneous flap virtually eliminates facial nerve palsy at the expense of a decreased exposure due to the bulky temporalis muscle ${ }^{11}$.

The surgical technique that we have described is easy to perform due to the obvious anatomical landmarks used (superficial temporal, temporalis muscle) and has not been associated, in our initial experience, with paralysis of the temporal branch of the facial nerve. In addition this surgical technique may provide wide exposure of the pterional-zygomatic arch region.

\section{Conclusion}

We submit this subfascial technique as a useful int junct to standard exposures of the pterional-zygomill fy arch region. Its advantages lie in its simple exoutiois 

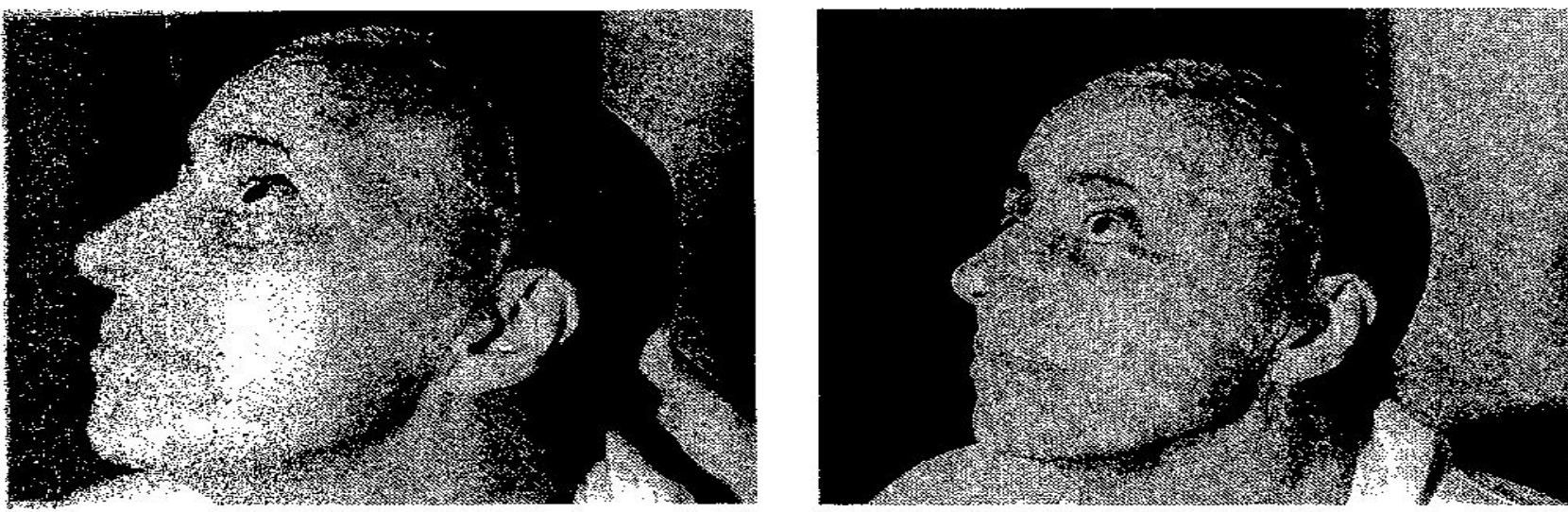

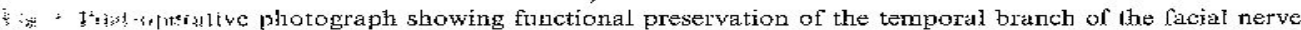

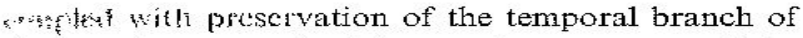
this fat inter and with extensive exposure of the

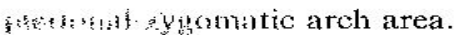

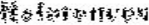

3: eilo (), Anand VK (1990) Zygomatic approach to skult-

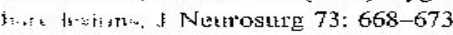

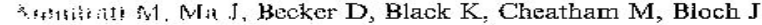

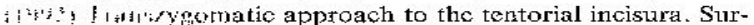

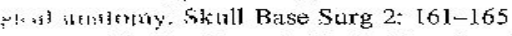

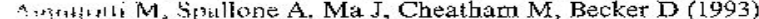

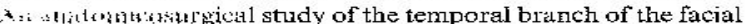

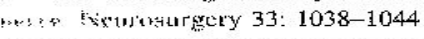

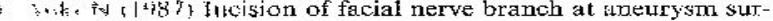

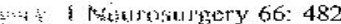

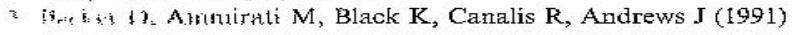
ftitiafy

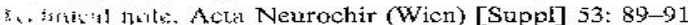

6. Fujitsu K, Kuwabara T (1985) Zygomalic approach for lesions in the interpeduncular cistern. J Neurosurg 62: 340-343

7. Gates GA (1988) The latcral facial approach to the nasopharymx and infratemporal fossa. Otalaryngol Head Neck Surg 99: 321325

8. Takuba A, Liu S, Nishimura S (1986) The orbitozygomatic infratemporal approach: an new surgical technique. Surg Neurol 26: $271-276$

9. Stuzin .JM, Wagstrom L, Kawamoto HK, Wolfe SA (1989) Anatony of the frontal branch of the fucial nerve: the significance of the temporal fat pad. Plast Reconsta Surg 83: 265-27

10. Yaşargil MG (1984) General operative techniques. In: Microneurosurgery, Vol 1. Thieme, New York

11. Yaşargil MG, Reichmann MV, Kubik S (1987) Preservation of the frontotemporal branch of the facial nerve using the interfascial temporalis flap for pterional craniotomy. J Newrosurg 67: 463-466

Correspondence: Mario Ammixati, M.I3., Division of Neurosurgery, Room 74-140 CHIS, University of California, 10833 Le Conte Avesue, Los Angeles, CA 90024-6901, U.S.A. 